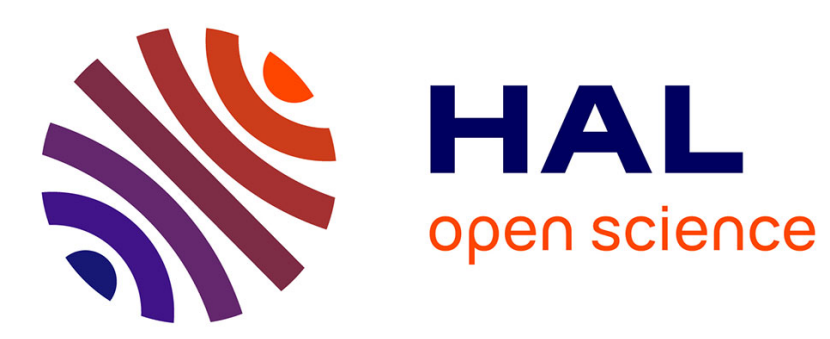

\title{
Conditionnel temporel objectif et constructions non téléonomiques en français et en serbe
}

Tijana Asic, Jacques Bres, Milana Dodig, Frédéric Torterat

\section{To cite this version:}

Tijana Asic, Jacques Bres, Milana Dodig, Frédéric Torterat. Conditionnel temporel objectif et constructions non téléonomiques en français et en serbe. Travaux de Linguistique: Revue Internationale de Linguistique Française, 2017, 75, pp.7-29. 10.3917/tl.075.0007 . hal-01705421

\section{HAL Id: hal-01705421 \\ https://hal.umontpellier.fr/hal-01705421}

Submitted on 28 Jun 2018

HAL is a multi-disciplinary open access archive for the deposit and dissemination of scientific research documents, whether they are published or not. The documents may come from teaching and research institutions in France or abroad, or from public or private research centers.
L'archive ouverte pluridisciplinaire HAL, est destinée au dépôt et à la diffusion de documents scientifiques de niveau recherche, publiés ou non, émanant des établissements d'enseignement et de recherche français ou étrangers, des laboratoires publics ou privés. 
*Asic T., Bres J., Dodig M. et Torterat F., 2017, «Conditionnel temporel objectif et tournures non téléonomiques en français et en serbe », Travaux de linguistique 75, 7-29.

\title{
Conditionnel temporel objectif et constructions non téléonomiques en français et en serbe
}

\begin{abstract}
Résumés
L'article analyse et compare trois constructions qui permettent de signifier, dans l'époque passée, la relation de progression temporelle effective entre un événement $\mathrm{e}_{1}$ et un événement $\mathrm{e}_{2}$, les deux premières en français, la troisième en serbe : $\mathrm{e}_{2}$ peut être actualisé au conditionnel objectif en français, ou intégré à une proposition subordonnée non téléonomique en français et en serbe.

Après une description de ces trois constructions à partir d'un corpus d'une centaine d'occurrences pour chacun d'eux, on calcule le taux de transpositions possibles de l'un à l'autre tour, puis on analyse les raisons qui bloquent les transferts : elles tiennent à la structure de chacune de ces constructions et aux contraintes d'ordre syntaxique, sémantique et énonciatif qu'elle leur impose. Il apparaît que certaines de ces contraintes peuvent être comprises comme des rémanences du tour originel dont chacune des trois constructions procède.
\end{abstract}

Mots clefs : progression, conditionnel, non téléonomique, traduction, grammaticalisation

\section{Objective temporal conditional and non teleonomic forms in French and Serbian}

This paper analyses and compares three constructions that enable to denote a relation of the effective temporal progression between an event $\mathrm{e}_{1}$ and an event $\mathrm{e}_{2}$, both situated in the past. The first two strategies concern French and the third one Serbian language. The event $e_{2}$ can be actualized in French via the so-called "objective" conditional or integrated into a non teleonomic subordinate clause in French and Serbian.

After a detailed description of these three constructions based on the 100 sentences corpus for each of them, we calculate the range of the possible transposition between them. Then we investigate the reasons that preclude the transfers: they are related to the structures of each of them and more precisely to the syntactic, semantic and pragmatic constraints. It seems that some of these constraints can be seen as remnants of the original form from which they have evolved.

Keywords: progression, conditional, non téléonomic, traduction, grammaticalization

\section{Introduction}

Comment se signifie, dans l'époque passée, la relation de progression temporelle effective entre un événement $\mathrm{e}_{1}$ et un événement $\mathrm{e}_{2}$, soit $\left[\mathrm{e}_{1}<\mathrm{e}_{2}\right]$, en français et en serbe ? Fréquemment, notamment en textualité narrative, la relation de progression temporelle se réalise par la simple succession des énoncés, représentant iconiquement la succession des événements (et complémentairement par des circonstants temporels venant appuyer des scripts) : 
[1] Margarèthe entra à Ravensbrück le 2 août $1940\left(\mathrm{e}_{1}\right)$. Elle n'en sortit pas avant avril $1945\left(\mathrm{e}_{2}\right)$.

[1a] Margareta je zatvorena u Ravensbrik 2.avgusta 1940.( $\left.\mathrm{e}_{1}\right)$. Iz njega je izašla tek aprila 1945. ( $\left.\mathrm{e}_{2}\right)$.

Aucun marquage syntaxique ni verbo-temporel de la progression, qui se signifie par la succession des deux procès, en appui sur les circonstants chroniques de datation « le 2 août 1940 » / «avril 1945 »: le procès de $e_{2}$ est au même temps du passé que celui de $\mathrm{e}_{1}$ (passé simple en français, passé composé en serbe).

Les langues ont trouvé cependant différentes possibilités pour signifier la progression $\left[\mathrm{e}_{1}<\mathrm{e}_{2}\right]$ syntaxiquement et par la morphologie verbale ; nous nous intéresserons ici seulement à trois d'entre elles, quelque peu marginales, les deux premières en français, la troisième en serbe, illustrées par les ex. [2, 2a, 2b] :

[2] Margarèthe entra à Ravensbrück le 2 août $1940\left(\mathrm{e}_{1}\right)$. Elle n'en sortirait pas avant avril $1945\left(\mathrm{e}_{2}\right)$ (Marie-Claire $\mathrm{n}^{\circ} 435$, novembre 1988)

[2a] Margarèthe entra à Ravensbrück le 2 août $1940\left(\mathrm{e}_{1}\right)$, pour n'en sortir qu'en avril $1945\left(\mathrm{e}_{2}\right)$.

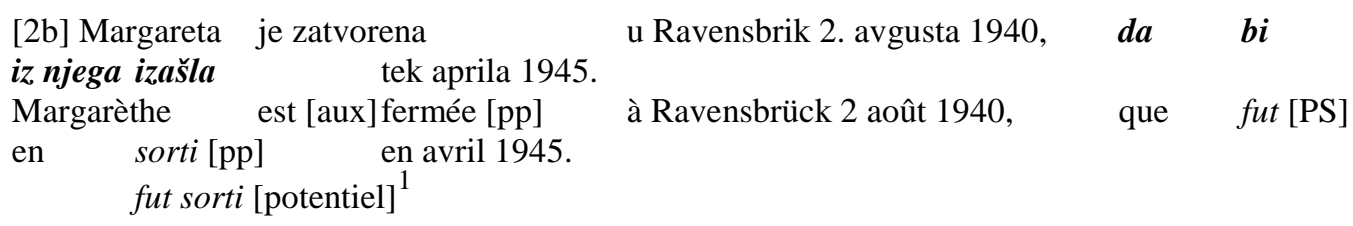

Pour actualiser le second procès (sortir),

- le français use en [2] du conditionnel (sortirait), ce que ne peut faire le serbe qui, s'il dispose d'un mode potentiel par lequel bien des emplois du conditionnel français peuvent trouver un équivalent, ne peut, dans le cas de [2], traduire, en indépendante, le conditionnel par un potentiel ${ }^{2}$;

- le français peut user, comme en [2a], d'une subordonnée finale non téléonomique à l'infinitif (pour en sortir) ;

- le serbe peut user également, comme en [2b], d'une subordonnée finale non téléonomique, mais au mode potentiel.

\footnotetext{
${ }^{1}$ Le potentiel se construit sur l'aoriste du verbe biti ('être') précédant le participe passé du verbe.

${ }^{2}$ Il le pourrait par un futur :

[2c] Margareta je zatvorena u Ravensbrik 2.avgusta $1940\left(\mathrm{e}_{1}\right)$. Iz njega neće izaći [FS, sortira] pre aprila $1945\left(\mathrm{e}_{2}\right)$.
}

Possibilité également réalisée par le français :

[2d] Margarèthe entra à Ravensbrück le 2 août $1940\left(\mathrm{e}_{1}\right)$. Elle n'en sortira pas avant avril $1945\left(\mathrm{e}_{2}\right)$.

\footnotetext{
${ }^{3}$ Nous parlons par la suite de téléonomique / non téléonomique plutôt que de finale /non finale : téléonomique présente des avantages pour une analyse des langues romanes et des langues slaves, en ce qu'il s'applique tant à la description du procès qu'à celle de la sémantique des opérateurs (cf. parmi d'autres Divjak, 2001 ; Araújo, 2009 ; Costa, 2010).
} 
Ces trois constructions, dans cet emploi que nous nommerons narratif, partagent plusieurs éléments :

- diachroniquement, elles n'apparaissent pas initialement mais au cours de l'histoire de la langue, et comme dérivation/grammaticalisation d'un fonctionnement antérieur, qui ne produit pas ce sens narratif, ce que nous développerons infra;

- le procès $e_{2}$ est dit et compris comme s'étant effectivement produit dans le passé, ultérieurement au procès $\mathrm{e}_{1}$. Ce que testent plusieurs faits, que nous illustrons sur la construction au conditionnel mais qui valent, mutatis mutandis, pour les tours non téléonomiques :

- $\mathrm{e}_{2}$ peut être mis au même temps du passé que $\mathrm{e}_{1}$ : sortirait en [2] peut être remplacé par sortit (cas de [1]), sans que cela change l'interprétation temporelle ;

$-\mathrm{e}_{2}$ ne peut faire l'objet d'une modalisation épistémique [2e] à la différence d'un énoncé au conditionnel en usage temporel standard [3] :

[2e] Margarèthe entra à Ravensbrück le 2 août $1940\left(\mathrm{e}_{1}\right)$. Elle n'en sortirait certainement pas avant avril $1945\left(\mathrm{e}_{2}\right)$.

[3] il fut d'avis qu'on était là plus en sûreté, personne ne viendrait certainement les chercher dans ce foin. (É. Zola, Germinal)

$-\mathrm{e}_{2}$ ne peut être suivi d'une négation du même procès [2f] à la différence d'un énoncé au conditionnel en usage temporel standard [4] :

[2f] Margarèthe entra à Ravensbrück le 2 août $1940\left(\mathrm{e}_{1}\right)$. Elle n'en sortirait pas avant avril $1945\left(\mathrm{e}_{2}\right)$. *Elle n'en sortit pas.

[4] Le soir Milady donna l'ordre d'introduire M. d'Artagnan aussitôt qu'il viendrait, selon son habitude. Mais il ne vint pas. (A. Dumas, Les Trois mousquetaires)

- sémantiquement: la progression temporelle entre $\mathrm{e}_{1}$ et $\mathrm{e}_{2}$ se signifie comme mise en perspective ultérieure de $\mathrm{e}_{2}$ par rapport à $\mathrm{e}_{1}$, effet de sens qui procède de la sémantique de leur emploi standard.

Ces trois constructions sont peu (pour [2] et [2a]), voire pas (pour [2b]), décrites dans les grammaires. Citons pour l'emploi objectif du conditionnel (désormais CO) : Damourette et Pichon1911-1936, Nilsson Ehle 1943, Martin 1971, Bres 2010, 2012 ; pour le tour non téléonomique français (désormais Pour + inf.), Cadiot 1991, 1997 et Colinet et al. 2014 ; le tour non téléonomique serbe, quant à lui, ne figure pas dans les grammaires, et n'a été mentionné qu'une seule fois dans un article (Popović 1977).

L'étude sera conduite à partir d'un corpus de 100 occurrences de chacune de ces trois constructions relevées dans la presse, la littérature, sur le net (et l'oral conversationnel lorsque le tour s'y voit réalisé).

Dans un premier temps, nous décrirons le fonctionnement de ces constructions qui permettent l'expression de la progression temporelle dans le passé (sections 1et 2). Dans un second temps, nous établirons les (im)possibles transferts /traductions de chacune dans les deux autres, ce qui permettra de préciser la sémantique et le fonctionnement textuel de chacune d'elles (section 3). 


\section{Conditionnel en emploi narratif}

\subsection{Du conditionnel subjectif au conditionnel objectif ${ }^{4}$}

Les premières occurrences de conditionnel objectif n'apparaissent qu'au XIX ${ }^{\mathrm{e}}$, soit donc quelque neuf siècles après la première occurrence attestée (pour l'heure) de conditionnel subjectif (Cantilène de sainte Eulalie, 880). Dès ses origines médiévales, le conditionnel a deux types d'emploi : temporel et modal. Le CO procède de l'emploi temporel, dans lequel le conditionnel est employé pour signifier l'ultériorité subjective, comme en [5] :

[5] Il pensa que la police ne serait pas longue à le reprendre, et on l'accuserait de rapt d'enfant. (J.-M. G. Le Clézio, Le Déluge)

Les procès être pas long à et accuser sont énoncés non directement par le narrateur, mais via la médiation énonciative du personnage masculin, très précisément sa pensée («il pensa que »). Conséquence de cette médiation: les procès au conditionnel sont posés comme ultérieurs à l'énonciation passée de l'actant $i l$, ultériorité dans le passé qui comme l'ultériorité dans le présent relève du temps ramifié (Gardies 1975) : les événements $e_{2}$ être pas long à et $\mathrm{e}_{3}$ accuser sont bien ultérieurs à $\mathrm{e}_{1}$ (pensa), mais cette ultériorité est seulement subjective. Les événements $\mathrm{e}_{2}$ et $\mathrm{e}_{3}$ ne sont pas inscrits dans l'unilinéarité du passé objectivement (et donc rétrospectivement par le narrateur), ils ne sont qu'envisagés par une énonciation du personnage comme ultérieurs, c'est-à-dire qu'ils s'inscrivent dans la ramification des possibles; le narrateur ne se prononce pas sur le fait qu'ils échoiront ou n'échoiront pas à la ligne du temps.

Ce n'est qu'au XIX ${ }^{\mathrm{e}}$ que le conditionnel sera à même de grammaticaliser l'ultériorité subjective en ultériorité objective, à savoir que $\mathrm{e}_{2}$ au conditionnel procèdera non de l'énonciation d'un personnage mais de celle du narrateur comme en [2], où l'événement $\mathrm{e}_{2}$ actualisé par le conditionnel ( «sortirait de Ravensbruck») sera inscrit dans l'unilinéarité du passé au même titre (mais pas de la même façon) que l'événement $\mathrm{e}_{1}$, entra, actualisé au PS. Sans retracer précisément ici le processus de grammaticalisation qui conduit du subjectif à l'objectif (Bres 2010, 2012), nous dirons que l'emploi objectif du conditionnel conserve l'ultériorité de l'emploi subjectif mais, selon un mécanisme d'abstraction/généralisation, efface son arrimage à une énonciation autre que celle du narrateur : $\mathrm{e}_{2}$ est simplement mis en perspective d'ultériorité à partir de $\mathrm{e}_{1}$, ou d'un élément de l'énoncé de $\mathrm{e}_{1}$.

Citons la première occurrence (1833) de notre corpus :

[6] Les germes de l'économie politique à l'usage du Parisien, latents en son coeur, ne devaient pas tarder à y fleurir, aussitôt que de spectateur oisif il deviendrait acteur dans le drame de la vie réelle. (Balzac, Eugénie Grandet) $(\approx \text { devint })^{5}$

\subsection{Les emplois syntaxiques et textuels du conditionnel objectif}

\footnotetext{
${ }^{4}$ Nous empruntons à Nilsson Ehle (1943) les termes de subjectif et d'objectif.

${ }^{5}$ Notons que cette grammaticalisation de l'ultériorité subjective en ultériorité objective ici analysée pour le conditionnel se réalise dès la fin du XVIII ${ }^{\mathrm{e}}$ pour aller et devoir à l'imparfait + inf. Il est significatif que dans [6] le CO deviendrait soit précédé de devaient tarder.
} 
Syntaxiquement, le conditionnel objectif apparaît en relative (36 oc. / 100), en circonstancielle (peu souvent) (2/100), en indépendante (62/100). On traitera ici presque exclusivement ces dernières. Le sujet de $e_{2}$ peut être coréférent à celui de $\mathrm{e}_{1}$ (supra [2]), ou différent de lui [8]. Nous ne distinguerons pas ces deux cas. On relève quatre types d'emplois :

(i) L'énoncé au $\mathrm{CO}$ est une simple prolepse : il n'est pas intégré à la diégèse. Le récit enchaîne ensuite sur $\mathrm{e}_{1}$ (et non sur $\mathrm{e}_{2}$ ); et de fait, du point de vue de la seule diégèse, on pourrait sauter cet énoncé, ou ces énoncés comme en [7] :

[7] Avant de partir, il se pencha vers sa femme, et lui dit $\left(\mathrm{e}_{1}\right)$ quelque chose. Etrangement elle ne se souviendrait $\left(\mathrm{e}_{2}\right)$ pas de ces mots. Leur dernier échange se volatiliserait $\left(\mathrm{e}_{2}\right)$. Et puis elle s'endormit $\left(\mathrm{e}_{3}\right)$. (D. Foenkinos, La Délicatesse $)$ [pencha $<\operatorname{dit}\left(\mathrm{e}_{1}\right)(<<<$ se souviendrait, se volatiliserait $)\left(\mathrm{e}_{2}\right)<$ s'endormit $\left.\left(\mathrm{e}_{3}\right)\right]$

Les deux énoncés au $\mathrm{CO}$ font faire au récit un bond en avant, de type parenthétique : le procès de l'énoncé qui suit, à savoir s'endormit $\left(\mathrm{e}_{3}\right)$, enchaîne temporellement non sur se souviendrait, se volatiliserait $\left(\mathrm{e}_{2}\right)$, mais sur dit $\left(\mathrm{e}_{1}\right)$.

(ii) L'énoncé au CO clôt un récit ou un épisode : il en actualise la dernière proposition narrative :

[8] Assassinat de la milliardaire Hélène Pastor: La garde à vue de son gendre validée

Le 6 mai 2014, en début de soirée, la milliardaire et son chauffeur étaient tombés dans un guet-apens devant l'hôpital Larchet, à Nice, [...]. L'employé Mohamed Darwich, 54 ans, avait succombé $\left(\mathrm{e}_{1}\right)$ à ses blessures le 10 mai. Helène Pastor, elle, mourrait $\left(\mathrm{e}_{2}\right)$ le 21 mai, à l'âge de 77 ans. (20 Minutes, 9/02/2016) [avait succombé $\left(\mathrm{e}_{1}\right)<$ mourrait $\left(\mathrm{e}_{2}\right)$ ]

(iii) L'énoncé au CO est intégré à la progression narrative ; il en est un chaînon :

[9] Cela fait, son bagage récupéré, Victoire choisit $\left(\mathrm{e}_{1}\right)$ un hôtel dans une rue qui n'aboutissait pas au port.

Elle n'y passerait $\left(\mathrm{e}_{2}\right)$ qu'une nuit. Le lendemain, scotchées sur les portes vitrées des commerces, elle examina $\left(\mathrm{e}_{3}\right)$ les annonces des particuliers. Elle trouva plutôt vite, en fin de matinée, une offre qui pourrait faire l'affaire. (J. Echenoz, Un an) [choisit $\left(\mathrm{e}_{1}\right)<$ passerait $\left.\left(\mathrm{e}_{2}\right)<\operatorname{examina}\left(\mathrm{e}_{3}\right)\right]$

Passerait $\left(\mathrm{e}_{2}\right)$ fait suite à choisit $\left(\mathrm{e}_{1}\right)$ et précède temporellement examina $\left(\mathrm{e}_{3}\right)$.

Dans les emplois (ii) et (iii), l'énoncé au $\mathrm{CO}$, du fait de son intégration à la diégèse, ne saurait être effacé.

Dans ces trois cotextes, le $\mathrm{CO}$, en prolepse, en clôture ou en chaînon narratif, est employé sporadiquement : il n'actualise qu'une, voire deux propositions, et cède immédiatement la place à un temps narratif plus prototypique. D'autre part, la distance temporelle entre $\mathrm{e}_{1}$ et $\mathrm{e}_{2}$ est significative : c'est bien sûr le cas de l'emploi proleptique [7], mais également des emplois de clôture (plusieurs jours en [8]) et de chaînon narratif (« une nuit » en [9]) : le conditionnel objectif semble se justifier de la mise en perspective d'ultériorité qu'il permet de réaliser. Ce qui, on va le voir, n'est pas le cas de l'emploi suivant. 
(iv) Nous avons relevé dans le texte littéraire contemporain, très précisément chez J. Echenoz, et dans une moindre mesure chez D. Foenkinos ou M. Houellebecq, un fonctionnement très marqué : le $\mathrm{CO}$ en rafales :

[10] Quand même on l'invita, parfois elle répondit $\left(\mathrm{e}_{1}\right)$. Les sujets britanniques organisaient le mardi des soirées passées à danser le cake-walk sur la terrasse en Adidas, en bermuda, en transpirant parmi les tables chargées de bouteilles. Un soir, un seul soir, Gloire se laisserait $\left(\mathrm{e}_{2}\right)$ aller à vider cinq ou six verres d'affilée.

Puis rentrerait $\left(\mathrm{e}_{3}\right)$ totalement ivre au Club. Mettrait $\left(\mathrm{e}_{4}\right)$ un temps fou à retrouver sa clef, puis la serrure, puis une fois entrée l'interrupteur de la veilleuse. Elle pousserait $\left(\mathrm{e}_{5}\right)$ un cri bref en croyant distinguer une petite forme en travers de son lit. Puis elle se reprendrait, se raisonnerait $\left(\mathrm{e}_{6}\right):$ ma pauvre vieille tu es encore complètement bourrée. . Mais non : au bruit de la porte claquée la petite forme se redressa $\left(\mathrm{e}_{7}\right)$ brusquement, l'air mauvais. (J. Echenoz, Les Grandes blondes) [répondit $\left(\mathrm{e}_{1}\right)<$ se laisserait aller $\left(e_{2}\right)<$ rentrerait $\left(e_{3}\right)<$ mettrait $\left(e_{4}\right)<$ pousserait $\left(e_{5}\right)<$ se reprendrait, se raisonnerait $\left(\mathrm{e}_{6}\right)<$ se redressa $\left.\left(\mathrm{e}_{7}\right)\right]$

Plusieurs procès, qui se succèdent immédiatement (ce que signale l'adverbe temporel puis), sont actualisés au conditionnel (se laisserait aller $\left(\mathrm{e}_{2}\right)$, rentrerait $\left(\mathrm{e}_{3}\right)<$ mettrait $\left(\mathrm{e}_{4}\right)$, pousserait $\left(\mathrm{e}_{5}\right)$, se reprendrait, se raisonnerait $\left(\mathrm{e}_{6}\right)$ ), avant de céder la place au passé simple (se redressa, $\left(\mathrm{e}_{7}\right)$ ).

Synthèse : la langue française a grammaticalisé le conditionnel subjectif en conditionnel objectif en effaçant l'énonciation rapportée à partir de laquelle le procès est posé comme ultérieur mais en conservant le sémantisme d'ultériorité. De la sorte, elle dispose d'un temps verbal pour signifier l'ultériorité objective dans le passé. Elle en use (i) pour construire localement une prolepse $\left[\mathrm{e}_{1}\left(\ll<<\boldsymbol{e}_{2}\right)<\mathrm{e}_{3}\right]$; (ii) pour intégrer sporadiquement un procès $\mathrm{e}_{2}$ ultérieur dans la trame narrative : $\left[\mathrm{e}_{1}<\boldsymbol{e}_{2}<\mathrm{e}_{3}\right]$; (iii) pour développer, sous forme de rafale, une séquence de procès, le plus souvent immédiatement successifs : $\left[\mathrm{e}_{1}<\boldsymbol{e}_{2}<\boldsymbol{e}_{3}<\boldsymbol{e}_{4}<\boldsymbol{e}_{5}<\right.$ $\left.\mathrm{e}_{6}\right]$.

Nous n'avons pas relevé d'occurrence de $\mathrm{CO}$ en interaction conversationnelle, notamment dans les récits oraux. Notre corpus fait état d'une seule occurrence en discours rapporté direct, dans une relative :

[11] Monsieur Tenant, qui, tout à ses souvenances, ne s'en rendit pas compte, arrêta Adélaïde, pour une fois pressée de s'éloigner : « [...] Venez, je vais vous montrer l'endroit où j'ai enfin osé dire, à la fille qui deviendrait ma femme, que je l'aimais. » Il l'entraînait, sans jamais hésiter sur le chemin à prendre, d'une galerie à l'autre. (H. Bianciotti, Sans la miséricorde du Christ)

L'usage du CO deviendrait en discours rapporté direct ne nous semble pas relever de la réalité de la pratique orale, mais être une projection d'un tour écrit.

Le CO, issu de pratiques scripturales, n'a pas à l'heure actuelle conquis les terres de l'oral conversationnel. Est-ce à dire pour autant qu'il relève du plan d'énonciation de l'histoire (Benveniste 1966 [1959]) ? On pourrait faire cette inférence à la lecture de la plupart des occurrences, qui effectivement appartiennent à ce type d'énonciation. Des exemples comme [12] nous en préviennent cependant :

[12] Marcel Habran est une figure de l'époque où les méchants n'étaient pas des barbus radicalisés. Lui, il a toujours porté beau : chemises, vestes pied-de-poule, chevelure soignée [...]. Il a été condamné une bonne douzaine de fois. La première, c'est en 1952, pour un vol de métaux à l'époque où il marnait 
comme manœuvre dans une usine - il deviendrait, par la suite, Julot casse-croûte avant de faire son trou dans le milieu de la prostitution. (Le Soir, 10/02/2016)

Les présents en emploi déictique (est, est cultivé, s'exprime) et les passés composés (a porté, a fait, a été condamné) inscrivent ce texte dans le plan d'énonciation du discours.

Notons que l'instruction d'ultériorité fournie par le CO s'appuie assez souvent $(54 \%$ des occurrences) sur une datation chronique ou sur un circonstant d'ultériorité (bientôt, plus tard, enfin, un jour, puis, par la suite, ensuite). On ne trouve par contre aucune occurrence d'adverbe (ou équivalent) localisant le procès déictiquement (hier, il y a trois jours, etc.). Nous reviendrons sur cette difficulté de conjoindre déictique et CO dans la section 3.

\section{Les non-téléonomiques en français et en serbe}

Les constructions étant largement parallèles en français et en serbe, nous les traiterons dans la même section. Il s'agit en effet dans les deux cas d'utiliser la structure de subordination circonstancielle téléonomique, pour un usage non téléonomique (désormais NT), très précisément temporel : signifier l'ultériorité dans le passé d'un procès $\mathrm{e}_{2}$ par rapport à un procès $\mathrm{e}_{1}$.

\subsection{La production de la construction}

\subsubsection{En français}

On sait qu'en ancien français por est susceptible de différentes acceptions, les deux principales étant d'introduire soit une relation de but («valeur prospective » (Buridant 2001 : 477) ou téléonomique), soit une relation de cause («valeur d'antécédence » (ibid.)). Le tour NT peut être analysé comme dérivé de la première. Comparons [13] et [14] :

[13] Dieudonné a habilement organisé son insolvabilité pour éviter de payer les quelque 65000 euros d'amende qui lui ont été infligés. (Le Monde, 07/01/2014)

[14] Marcel Saez, chauffeur de taxi, n'est pas prêt d'oublier sa course du 31 décembre... Une course entamée à l'aube, $3 \mathrm{~h} 35$, pour se terminer presque deux heures plus tard à l'hôtel de police... où sa cliente a avoué le meurtre de l'homme qui lui offrait l'hospitalité. (Est Républicain, 01-02-2003)

En [13], le procès éviter $\left(\mathrm{e}_{2}\right)$ est un but procédant de l'intention du sujet de $\left(\mathrm{e}_{1}\right)$ (organiser). En tant que prospectif, ce procès s'inscrit dans l'ultériorité ramifiée de l'intention du sujet +humain et non dans l'unilinéarité objective du passé : ce but pourra par la suite s'avérer s'être réalisé ou ne pas s'être réalisé. Cet énoncé accepte aussi bien la suite : «et effectivement il n'a pas payé cette amende », que «mais le stratagème a échoué, et il a dû payer cette amende ».

En [14], le procès se terminer $\left(\mathrm{e}_{2}\right)$ ne procède pas de l'intention du sujet de « entamée » $\left(\mathrm{e}_{1}\right)$, à savoir «une course ». Il est posé comme s'étant effectivement réalisé, ultérieurement à $\left(\mathrm{e}_{1}\right)$ («presque deux heures plus tard »). Du tour téléonomique au tour non téléonomique : (i) l'élément intentionnel est effacé : en conséquence, le sujet de $\left(\mathrm{e}_{1}\right)$ qui est également le contrôleur (implicite) de l'infinitif $\left(\mathrm{e}_{2}\right)$, ne doit plus avoir forcément le trait +humain (cf. «la course » en [14]); (ii) l'élément ultériorité, qui accompagnait implicitement l'intention, devient essentiel. $\left(\mathrm{e}_{2}\right)$, ne procédant plus de l'intention du sujet, est inscrit dans l'unilinéarité effective du passé $\left[\mathrm{e}_{1}\right.$ (entamer) $<\mathrm{e}_{2}$ (se terminer $\left.)\right]$. 
La construction étudiée ne saurait procéder du pour introduisant une relation de cause : l'infinitif dans ce cas est toujours à la forme composée [15], alors qu'il est à la forme simple dans la construction NT :

[15] En juin, trois jeunes membres de cette communauté ont été condamnés à de la prison à vie pour avoir organisé une filière de recrutement au profit de l'EI. (Le Monde, 1/12/2016)

Nous ne sommes pas en mesure de dater avec précision les étapes qui ont conduit du tour téléonomique au tour NT. Pour l'heure, à partir de nos premières recherches, nous pouvons avancer :

- Que dès le XII ${ }^{\mathrm{e}}$, on trouve des occurrences de pour + inf. comme :

[16] Miex nous vient il [...] que nous conquestons viande et avoir par raisnavle acoison, que nous i aillons pour morir de fain. (Robert de Clari, La Conqueste de Constantinople) ('Il est plus opportun pour nous d'acquérir des vivres et des biens de manière légitime, plutôt que d'y aller pour y mourir de faim')

dans lesquelles pour introduit «la simple conséquence prévisible d'un procès » (Moignet 1988 : 320). Si le procès $e_{2}$ (mourir de faim) ne procède pas de l'intention du sujet (nous), il n'est cependant pas inscrit dans la réalité du passé : le tour est syntaxiquement subordonné à une évaluation («miex nous vient il ») qui compare deux possibilités.

- Que, au début du XVII ${ }^{\mathrm{e}}$, on trouve des occurrences de pour + inf. NT, mais appliqué à des descriptions d'action au présent ou au futur, dans des textes scientifiques :

[17] Les artères portent le sang vital qui sort du ventricule gauche du coeur, pour ensuite estre porté à toutes les parties du corps comme leur nourriture, au lieu que les veines recoivent le sang qui reste de la nouriture des parties (François Bernier, Abrégé de la philosophie de Gassendi, 1684)

Il faut, semble-t-il, attendre la fin du XVII ${ }^{\mathrm{e}}$ pour relever les premières occurrences du tour signifiant l'ultériorité objective dans le passé, en textualité narrative :

[18] C'est ainsi qu'il a fait, et il a commencé par aimer son père pour ensuite aimer ce qu'il commandoit, quelque rigoureux qu'il parust à la nature, car l'amour de celuy qui commande rend doux ce qui est amer et rude. Aimons donc Jésus-Christ (J.-B. Bossuet, Méditations sur l'Évangile, 1704)

La construction se développe au $\mathrm{XX}^{\mathrm{e}}$, notamment en textualité littéraire [19] et journalistique (supra [14]) :

[19] Je le savais, déclara Seven. Cette fille de qualité m'attendait. J'ai navigué vingt ans pour finalement échouer ici et convoler en justes noces avec elle. Messieurs, je vous invite, vous serez tous de la cérémonie. (Pierre Mac Orlan, À bord de l'étoile matutine)

Notons que pour, dans la construction NT, ne peut être remplacé par les locutions prépositives de sorte à, afin de ou en vue de [14a], avec lesquelles il est, en emploi téléonomique [13a], interchangeable :

[14a] Une course entamée à l'aube, 3h35, *afin de / *en vue de finalement se terminer presque deux heures plus tard à l'hôtel de police... 
[13a] Dieudonné a habilement organisé son insolvabilité afin de / en vue d'éviter de payer les quelque 65000 euros d'amende qui lui ont été infligés.

\subsubsection{En serbe}

Le serbe dispose de constructions téléonomiques comme [20]:

$\begin{array}{lllll}\text { [20 ] Upisao se u } & \text { školu plesa } & d a & \text { bi naučio } & \text { da igra tango. (Politika, } \\ \text { 13.01.2009.) } & & & & \\ \text { S'est inscrit dans école de danse } & \text { que fut appris (potentiel) } & \text { que danse tango. }\end{array}$

La fonction de la subordonnée est d'expliciter le but qui motive l'activité de l'agent dans la principale. En tant que but à atteindre, le procès apprendre le tango est de l'ordre du prospectif non-réalisé. Il est introduit par la conjonction $d a$ bi et est actualisé au potentiel morphologiquement : passé simple de l'auxiliaire être + participe passé du verbe - dont la fonction de base est d'actualiser un procès seulement envisagé.

Le tour NT $d a+$ potentiel se réalise de la même façon, mais produit le sens temporel d'ultériorité dans le passé :

[21] Ciceron iz Rima odlazi u Brindizi, a potom boravi sedam meseci u Solunu, da bi u jesen stigao u Drač. (Mostovi, № 103)

Cicéron de Rome part à Brindisi, et puis passe sept mois à Thessalonique, que fut à l'automne, arrivé à Durres.

Cicéron part de Rome à Brindisi, et puis passe un séjour de sept mois à Thessalonique, pour, à

l'automne, arriver à Durres.

Comme dans le tour français, l'élément d'intention est effacé au profit du seul élément d'ultériorité qui était impliqué (un but est toujours ultérieur à sa conception), cet effacement entraînant le remplacement de la ramification des possibles par l'unilinéarité du passé. Cicéron est dit arriver effectivement à Durres après avoir séjourné à Thessalonique : [ $\mathrm{e}_{1}$ (passer un séjour) $<\mathrm{e}_{2}$ (arriver à Durres)]. Le potentiel actualise le procès arriver non comme envisagé intentionnellement mais comme réalisé.

L'intention étant écartée, le sujet des NT $d a+$ potentiel n'est plus obligatoirement thumain : il peut s'agir d'un non -animé [22], voire d'un impersonnel [23]:

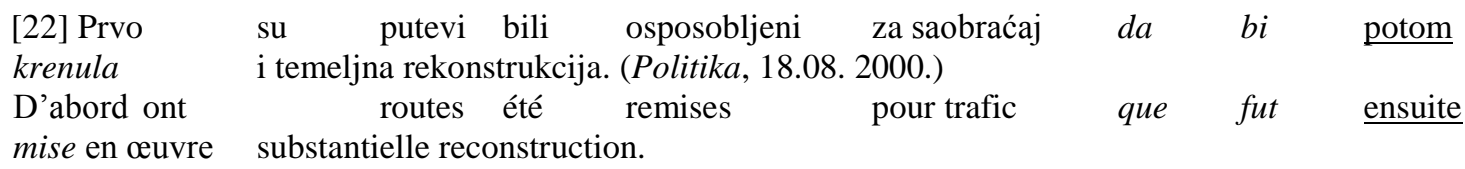

Les routes ont d'abord été remises en état de service et ensuite une reconstruction substantielle fut mise en œuvre.
[23] Ceo dan
je grmelo
da $b i \quad$ tek uveče
počela da pada kiša
Toute la journée il tonnait
que fut le soir commencé tomber pluie.

Il tonnait toute la journée et la pluie ne commença que le soir.

Notre hypothèse est que les NT en serbe procèdent, comme en français, des téléonomiques : nous ne disposons pas de matériau historique qui permette de l'étayer, ni ne pouvons dater précisément l'apparition du tour NT. Tout au plus pouvons-nous avancer qu'il est 
relativement récent, dans la mesure où, à l'exception de l'article de Popović (1977), il ne se voit pas mentionné dans les travaux sur le serbe.

\subsection{Les emplois syntaxiques et textuels de la construction non téléonomique}

Le fonctionnement temporel du tour NT, tant en français qu'en serbe, se vérifie des deux faits suivants :

- dans les deux langues, il peut être remplacé par avant de + inf. :

[19b] J'ai navigué vingt ans avant d'échouer ici et de convoler en justes noces avec elle.

[22a] Putevi su bili osposobljeni za saobraćaj pre nego što je krenula temeljna rekonstrukcija.

Routes ont été remises pour trafic avant que est mise en œuvre substantielle reconstruction.

Les routes ont été remises en état de service avant qu'une reconstruction substantielle ne fût mise en œuvre.

- à la différence du tour téléonomique, la subordonnée ne saurait être antéposée, la succession principale / subordonnée mimant la succession $\left[\mathrm{e}_{1}<\mathrm{e}_{2}\right]$.

$\begin{array}{cccc}\text { [23a] } & * \text { da bi tek uveče } & \text { počela da pada kiša } \quad \text { Ceo dan } & \text { je grmelo } \\ \text { que fut le soir } & \text { commencé tomber pluie, toute la journée } & \text { il tonnait }\end{array}$

En français, anticiper la subordonnée réintroduit au mieux, avec un sujet thumain, l'intentionalité, et donc fait de pour + inf., un énoncé téléonomique :

[19c] Pour finalement échouer ici et convoler en justes noces avec elle, j'ai navigué vingt ans.

Lorsque le sujet de la principale est +humain, à savoir doté d'intentions possibles, il peut y avoir ambiguïté entre interprétation téléonomique et interprétation NT :

[24] Après son retour à Joncherey, Marcel entra à la SID (Société industrielle Delle), pour y faire carrière au bureau des achats et au contrôle des factures où il fut très apprécié jusqu'à la préretraite, à 60 ans, en 1975. (Est Républicain, 20-01-2003)

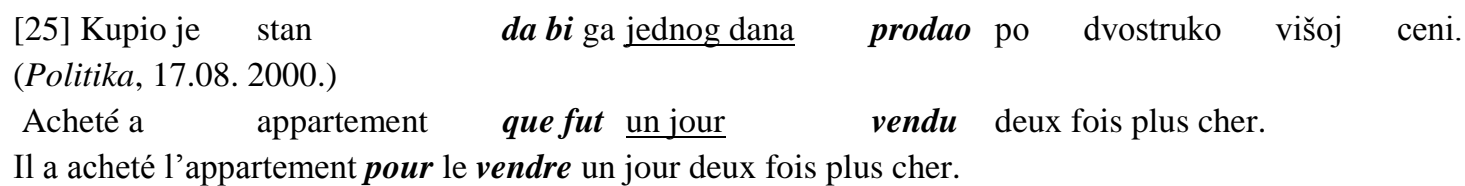

L'interprétation préférentielle de [24] est NT: Marcel semble bien être entré à la SID et y avoir fait ensuite carrière au bureau des achats, notamment du fait de la relative « où il fut très apprécié » qui présuppose qu'il a fait effectivement carrière dans ledit bureau - et non dans l'intention $d$ 'y faire carrière, encore que cette interprétation ne soit pas absolument exclue. L'interprétation préférentielle de [25] est, quant à elle, téléonomique : l'actant semble bien avoir acheté un appartement dans l'intention de doubler son prix à la revente, principalement du fait du circonstant temporel indéfini un jour ; mais une interprétation NT n'est cependant pas totalement écartée. 
C'est, nous semble-t-il, pour conjurer ce risque d'ambiguïté que :

- dans la plupart des cas ( $90 \%$ en français comme en serbe), la subordonnée contient un adverbe du type de ensuite, ou finalement, qui tend à inscrire le procès $\mathrm{e}_{2}$ comme ultérieur effectif.

- dans plus de la moitié des cas, le verbe de la subordonnée se présente comme une suite contraire à ce qui était logiquement attendu, ce qui écarte l'interprétation téléonomique qui présuppose la congruence entre $\mathrm{e}_{1}$ et $\mathrm{e}_{2}$ :

[26] « J'ai été reçu par un dirigeant de la Banque Publique d'investissement (BPI), raconte-t-il. Il s'est assis à l'écart et a passé son temps à envoyer des SMS pendant ma présentation, pour m'annoncer en fin de réunion qu'il ne comprenait pas ce que je faisais là », fulmine l'ancien capitaine, dont le caractère impétueux s'accorde mal avec l'ambiance feutrée des cabinets ministériels. (L'Expansion, 0907-2014)

[27] Boža Ilić je stvorio monumentalno delo, $\quad \boldsymbol{d a} \boldsymbol{b i}$ uprkos tome bio gotovo sklonjen iz umetničkog života. (Politika, 25.08.2000.)

Boža Ilić a créé monumentale œuvre, que fut pourtant été pratiquement éliminé de artistique vie.

pour serait éliminé

Boža Ilić a créé une œuvre monumentale, pour être pourtant pratiquement éliminé de la vie artistique.

La principale soulève une expectative logique qui se voit contrariée par la suite chronologique fournie par la subordonnée : en (27) p. ex., la mise à l'écart de Boža Ilić $\left(\mathrm{e}_{2}\right)$ est contraire à ce qui pouvait être inféré de ce qu'il a produit une « œuvre monumentale » $\left(\mathrm{e}_{1}\right)$.

Textuellement, la construction NT apparaît majoritairement ( $87 \%$ en français, $75 \%$ en serbe) pour actualiser le dernier procès d'une suite narrative : il en forme comme la coda, sur laquelle il focalise, tout particulièrement lorsque $\mathrm{e}_{2}$ s'oppose à ce qui était logiquement attendu, et / ou lorsque la subordonnée est détachée de la principale dans la structure syntaxique d'ajout après le point :

[28] Pour lui qui a beaucoup «poireauté » dans la file de départ de cette troisième montée, l'attente a été très longue. Pour finalement s'entendre dire que les voitures « ouvertes » ne monteraient pas une troisième fois. "On nous a annoncé qu'il n'y aurait pas de $3^{\text {e }}$ montée pour nous, pas de vainqueur final, mais que des vainqueurs de groupes et classes. C'est la première fois que cela se produit !» (L'Yonne, 30-09-2013)

[29] Prišao sam mu i opomenuo ga da bar malo smanji radio, što je on zakratko i učinio. Da bi posle dva tri pređena kilometra, valjda za inat, toliko pojačao radio da je pravo čudo kako nismo poiskakali iz auta. (Politika, 07.04.2001.)

Ai approché lui et demandé le que un peu baisse radio, ce qu'il a pour un instant fait. Que fut après deux trois kilomètres, probablement pour contrarier, tellement augmenté radio que est vrai miracle comment n'avons pas quitté le véhicule.

Je l'ai approché et lui ai demandé de baisser la radio, ne serait-ce qu'un peu, ce qu'il a fait pour un instant. Pour ensuite augmenter le volume, après deux ou trois kilomètres, probablement pour me contrarier, à tel point que c'est un vrai miracle si nous nous n'avons pas quitté le véhicule en catastrophe.

Quasi identiques sémantiquement, les deux tours NT, du fait de la différence d'actualisation $\mathrm{du}$ procès $\mathrm{e}_{2}$ - à l'infinitif pour le français, au potentiel pour le serbe -, diffèrent syntaxiquement sur deux points : 
- En français, l'infinitif de $\mathrm{e}_{2}$ demande que son contrôleur (implicite) soit co-référent avec le sujet de $\mathrm{e}_{1}$ : en (28) p. ex. c'est le dirigeant de la Banque Publique qui à la fois passe son temps à envoyer des SMS $\left(\mathrm{e}_{1}\right)$ et annonce en fin de réunion que... $\left(\mathrm{e}_{2}\right)$. En serbe, du fait que le verbe de $e_{2}$ est conjugué au potentiel, le tour n'est pas soumis à cette contrainte : le sujet de $e_{2}$ peut être co-référent à celui de $e_{1}$ [27], ou différent de lui [23]. Cette contrainte qui pèse sur $\mathrm{e}_{2}$ en français nous semble être à l'origine d'un trait syntaxique manifeste : le taux élevé de passifs $(18,6 \%$ en français, contre $1 \%$ en serbe), périphrastiques (être + participe passé) [30] ou pronominaux (se faire + infinitif) [31] :

[30] Ils devaient être déchargés à Saint-Nazaire il y a plusieurs jours déjà. Ils sont restés à bord de leur bateau de transport. Pour finalement être réexpédiés hier vers la Norvège. (Est Républicain, 19-022003)

[31] Elle a alerté la GRC pour finalement se faire dire que les enquêteurs ne pouvaient rien faire tant que son fraudeur ne lui demandait pas d'argent. «Parfait, se dit-elle, je vais aller jusqu'au bout. Cela pourrait aider d'autres personnes! ». (Le Journal de Montréal, 03-01-2015)

Le taux relativement élevé de passifs de $\mathrm{e}_{2}$ nous semble procéder de ce que cette diathèse permet de poser en contrôleur le deuxième actant du procès lorsque c'est nécessaire pour établir la coréférence avec le sujet de $\mathrm{e}_{1}$, ce qui autorise l'emploi de pour + inf.

- L'infinitif étant un mode atemporel, le tour pour + inf. peut apparaître non seulement pour des faits passés, mais également pour des faits présents, futurs, ou hypothétiques (8\% des cas) :

[32] Ici, la plateforme de l'enseigne de produits dégriffés, la seule en Limousin, recevra des produits en provenance du monde entier, pour ensuite les expédier vers les magasins du centre de la France. (Le Populaire du Centre, 08-02-2016)

[33] « On a toujours des plus gros scores quand on manifeste le samedi ou le dimanche. Tout comme on a des plus grosses manifestations quand il fait beau. Là, il pleuvait donc je pense que pas mal de gens ce sont découragés », indique-t-elle. «De toute façon, cela ne sert à rien de commencer hyper fort si c'est pour ensuite s'éteindre, juge-t-elle encore. Je pense que ce n'est que le début ». (BFMTV, « Manifestations contre la loi Travail »10.3.2016)

Le serbe n'utilisera pas $d a+$ potentiel dans ce type de structures (mais le futur dans la plupart des cas).

Si le conditionnel objectif comme la construction NT en français et en serbe permettent de construire une relation de progression temporelle effective entre un événement $\mathrm{e}_{1}$ et un événement $\mathrm{e}_{2}$ dans le passé, soit $\left[\mathrm{e}_{1}<\mathrm{e}_{2}\right]$, leurs conditions d'emploi sont en partie différentes, ce qui se manifeste par les impossibilités de remplacement ou de traduction de l'un par l'autre tour, comme nous allons le voir.

\section{Transpositions (im)possibles}

Contrairement à ce qu'on aurait pu inférer de l'exemple initial (2-2b), $\mathrm{e}_{2}$ dans les séquences $\left[\mathrm{e}_{1}<\mathrm{e}_{2}\right]$ n'est pas susceptible d'être actualisé indifféremment par l'une ou l'autre construction.

Le tableau suivant explicite les pourcentages de transpositions possibles (fondées sur les jugements de grammaticalité formulés par les auteurs de cet article) : en ordonnée, les occurrences où $e_{2}$ est actualisé, dans le corpus d'origine, soit au conditionnel objectif, soit par 
le tour pour + inf., soit par le tour $d a+$ potentiel ; en abscisse, le pourcentage de transpositions possibles dans les deux autres tours :

\begin{tabular}{|l|c|c|c|}
\hline & conditionnel & pour + infinitif & $d a+$ potentiel \\
\hline conditionnel & & $13 \%$ & $37 \%$ \\
\hline pour + infinitif & $86 \%$ & & $92 \%$ \\
\hline$d a+$ potentiel & $75 \%$ & $77 \%$ & \\
\hline
\end{tabular}

Les occurrences du corpus conditionnel sont transposables pour 13\% d'entre elles en pour + inf., et pour $37 \%$ en $d a+$ potentiel ; celles du corpus pour + inf. sont transposables pour $86 \%$ d'entre elles en conditionnel, et pour $92 \%$ d'entre elles en $d a+$ potentiel ; et celles du corpus $d a+$ potentiel sont transposables pour $75 \%$ d'entre elles en conditionnel, et pour $77 \%$ d'entre elles en pour + inf.

Première évidence :

- les deux tours NT ont des résultats fort similaires : ils ont des taux de transposition possible entre eux élevés : pour + inf. est traduisible par $d a+$ potentiel dans $92 \%$ des cas, et $d a+$ potentiel est traduisible par pour + inf. dans $77 \%$ des cas ; ils sont par ailleurs assez réticents à la transposition des tours au CO: seulement dans $13 \%$ des cas pour pour + inf. et $37 \%$ pour $d a+$ potentiel. On peut rendre compte de ces faits par la proximité syntaxique des deux constructions NT et par leur différence de structure avec le tour au CO ;

- le tour au CO, s'il est donc faiblement traductible par les tours NT, peut en revanche les transposer dans un grand nombre de cas : $86 \%$ pour pour + inf. et $75 \%$ pour $d a+$ potentiel. Il apparaît donc comme celui qui a le plus de puissance et le moins de contraintes pour signifier la progression temporelle $\left[\mathrm{e}_{1}<\mathrm{e}_{2}\right]$ dans le passé.

Explicitons les impossibilités de transposition : elles tiennent à la structure de chacune de ces constructions et aux contraintes qu'elles leur imposent. Ces contraintes sont d'ordre syntaxique (i, ii, vi), sémantique (iii, iv, v, vii) et énonciatif (viii) :

(i) Le tour au CO, de par sa liberté syntaxique - on le trouve majoritairement en indépendante - peut affecter successivement deux procès, voire fonctionner « en rafale » $\mathrm{e}_{1}$ $<\mathrm{CO} \mathrm{e}_{2}<\mathrm{CO} \mathrm{e}_{3}$, etc. ] (supra [10]). Il en va différemment des tours NT: en tant que propositions subordonnées, dépendant de la principale qui actualise $\mathrm{e}_{1}$, le tour est difficilement récursif. Et de fait nous n'avons relevé aucune occurrence qui enchaînerait à plusieurs reprises pour + inf. ou $d a+$ potentiel selon la structure: $\left[\mathrm{e}_{1}<\right.$ pour / da bi $\mathrm{e}_{2}<$ pour/da bi $e_{3}$, etc. ]. Les tours NT ne peuvent traduire les $\mathrm{CO}$ en rafales.

(ii) au $\mathrm{CO}, \mathrm{e}_{2}$ peut être inclus dans une relative périphrastique complément direct de $\mathrm{e}_{1}$ [34] ou complément de nom d'un SN complément de $e_{1}$ [35], donc faisant partie du SV construit autour de $\mathrm{e}_{1}$ : 
[34] Avec ce bougre-là, mon Esternome découvrit ce qui deviendrait son métier : art de nouer les poutrelles sans y mettre un seul clou, art d'équilibre des masses et de balance des poids, art du calcul des justes inclinaisons. (P. Chamoiseau, Texaco)

[35] C'est ainsi que, à chaque crise politique, à chaque rénovation artistique, Mme Verdurin avait arraché petit à petit, comme l'oiseau fait son nid, les bribes successives, provisoirement inutilisables, de ce qui serait un jour son salon. L'affaire Dreyfus avait passé, Anatole France lui restait. (M. Proust, À la recherche du temps perdu)

En [34] « ce qui deviendrait son métier » $\left(\mathrm{e}_{2}\right)$ est c.o.d. de « découvrit » $\left(\mathrm{e}_{1}\right)$; en [35], « de ce qui serait un jour son salon » est complément du $\mathrm{SN}$ « les bribes », c.o.d. de « avait arraché ». Ces occurrences ne sont pas transposables en NT qui demandent que les deux procès $e_{1}$ et $e_{2}$ ne fassent pas partie du même SV. Cette différence de fonctionnement s'explique par rémanence de la construction à l'origine de chacun d'eux : les procès au conditionnel subjectif appartiennent à des propositions subordonnées complétives ou interrogatives indirectes, c'està-dire relevant du SV. À l'inverse, les téléonomiques relèvent d'un SP de phrase. Conséquence de cela : le $\mathrm{CO}$ peut actualiser un procès $\mathrm{e}_{2}$ dans un syntagme relevant du $\mathrm{SV}$ de $\mathrm{e}_{1}$; les NT ne sauraient le faire : elles ne pourront donc pas transposer les $\mathrm{CO}$ de ce type de relative.

(iii) Avec le $\mathrm{CO}, \mathrm{e}_{2}$ peut n'avoir avec $\mathrm{e}_{1}$ qu'une relation temporelle de pure ultériorité, comme en (36) :

[36] À table il avait pensé lui plaire. Tandis que maintenant il ne savait plus. Plus tard il dirait : « Je sentais que vous n'étiez pas prête ». (A. Ferney, La Conversation amoureuse)

Alors même qu'il y a coréférence des sujets (cf. infra vi) et circonstant d'ultériorité (cf. infra iv) (plus tard), on ne saurait remplacer le CO dirait ni par pour inf. ni par da + potentiel :

[36a] A table il avait pensé lui plaire. Tandis que maintenant il ne savait plus, *pour plus tard dire : «Je sentais que vous n'étiez pas prête ».

Za stolom mislio je da je zadovolji.Dok sada nije znao više, $\quad$ *da kasnije kaže : "Osetio sam da niste spremni."

[36b] Mislio je za stolom da je zadovolji. Ali sada više nije znao, *da bi kasnije rekao : "Osetio sam da niste spremni."

que fut dit

C'est que les NT tendent à demander que la relation temporelle soit sous-tendue par une forme de relation logique, en particulier de conséquence inattendue nous l'avons vu, à tout le moins que les deux procès $e_{1}$ et $e_{2}$ appartiennent au même champ lexical comme $p$. ex. celui du voyage en [21]. Or en [36], il n'y a aucune relation autre que d'ultériorité entre savoir $\left(\mathrm{e}_{1}\right)$ et dire $\left(\mathrm{e}_{2}\right)$. Nous mettons cette différence au compte de la rémanence du sens originel : le conditionnel subjectif (cf. [4] supra) ne pose entre le verbe d'énonciation et le procès qu'il subordonne d'autre lien que celui d'ultériorité ; à l'inverse de pour et de $d a$ bi téléonomiques qui posent une relation de finalité. Si la finalité du tour téléonomique est effacée dans les NT, persiste l'idée d'un lien logique, à tout le moins sémantique comme en [21], autre que simplement chronologique, entre $\mathrm{e}_{1}$ et $\mathrm{e}_{2}$. 
(iv) certaines occurrences de $\mathrm{CO}$, dans lesquelles le conditionnel n'est pas accompagné d'un circonstant d'ultériorité, comme :

[37] Présenté chez Gallimard, l'ouvrage (Histoire de la folie) fut refusé. Il serait publié à l'automne aux éditions Plon. (E. Roudinesco, Philosophes dans la tourmente)

sont transposables en tour NT :

[37a] Présenté chez Gallimard, l'ouvrage (Histoire de la folie)

fut refusé, pour être

publié à l'automne aux éditions Plon.

[37b] Predstavljeno Galimaru, delo (Istorija ludila)

bilo je odbijeno, $d \boldsymbol{a} \quad \boldsymbol{b i} \quad \boldsymbol{b i l o}$

objavljeno na jesen u okviru Plonovih izdanja.

Présenté chez Gallimard,ouvrage (Histoire de la folie)

publié à l'automne aux éditions Plon.

a été refusé, que fut été

Ces transpositions sont nettement améliorées si on ajoute l'adverbe temporel ensuite :

[37c] Présenté chez Gallimard, l'ouvrage (Histoire de la folie) fut refusé, pour être ensuite publié à l'automne aux éditions Plon.

[37d] Predstavljeno Galimaru, delo (Istorija ludila) $\quad$ bilo je odbijeno, da $\quad$ bi $\quad$ potom $\quad$ bilo objavljeno na jesen u okviru Plonovih izdanja.

Présenté chez Gallimard,ouvrage (Histoire de la folie) a été refusé, que fut ensuite été publié à l'automne aux éditions Plon.

Nous mettrons en relation cette amélioration procurée par la présence de l'adverbe d'ultériorité avec le fait que, nous l'avons vu, les NT usent de ce type d'adverbe dans $90 \%$ des cas, contre seulement $54 \%$ dans le tour CO. Cette différence nous semble signaler que la mise en perspective d'ultériorité est plus forte pour le CO que pour les NT, qui ont souvent besoin de la béquille de l'adverbe pour la signifier clairement. Ce qui s'explique là aussi par rémanence du sens originel: le conditionnel, de par sa formation morphologique - le morphème -R- qu'il partage avec le futur - signifie basiquement l'ultériorité ; alors que cette notion ne fait qu'accompagner la téléonomie : pour présuppose seulement l'ultériorité du procès qu'il introduit.

(v) si l'ordre référentiel est $\left[\mathrm{e}_{1}<\mathrm{e}_{2}\right]$, l'ordre textuel, avec un $\mathrm{CO}$, peut présenter $\mathrm{e}_{2}$ avant $\mathrm{e}_{1}$ dans certaines relatives :

[38] Mais la gauche, qui ne tarderait $\left(\mathrm{e}_{2}\right)$ pas à devenir ouvertement antidynastique, n'avait su aucun gré à Louis XVIII de sa politique d'union nationale et elle se détacha bientôt du centre $\left(\mathrm{e}_{1}\right)$. (J. Bainville, Histoire de France)

Cette anticipation est interdite aux tours NT qui doivent obligatoirement présenter les événements $\mathrm{e}_{1}$ et $\mathrm{e}_{2}$ selon l'ordre référentiel : si pour + inf. ou da + potentiel est anticipé, il produit forcément la valeur téléonomique. Et remarquons que [38] n'est transposable en NT que si l'on déplace l'énoncé $e_{2}$ de la relative en fin de phrase :

[38a] Mais la gauche n'avait su aucun gré à Louis XVIII de sa politique d'union nationale et elle se détacha bientôt du centre $\left(\mathrm{e}_{1}\right)$ pour ne pas tarder à devenir ouvertement antidynastique $\left(\mathrm{e}_{2}\right)$.

[38b] Ali levica nije odavala nikakvu zahvalnost Luju XVIII za njegovu politiku o nacionalnom jedinstvu i ubrzo se odvojila od centra $\quad d a \quad b i$ otvoreno postala antidinastijska. 


\begin{abstract}
Mais la gauche n'avait su aucun gré à Louis XVIII pour sa politique de nationale union et bientôt se détacha du centre que fut ouvertement devenu antidynastique.
\end{abstract}

Ces différentes explications nous semblent rendre compte tant de la réticence de transposition des tours CO en NT (seulement $13 \%$ pour pour + inf. et $37 \%$ pour da + potentiel), comme à l'inverse du taux élevé de transpositions possibles des tours NT en CO $(86 \%$ pour pour + inf. et $75 \%$ pour $d a+$ potentiel).

D'autre part, les différences dans les pourcentages de transpositions entre les deux NT tiennent aux deux faits suivants :

(vi) nous l'avons vu (supra 2.2.), au CO comme dans $d a+$ potentiel, le sujet de $\mathrm{e}_{2}$ peut être co-référent ou différent de celui de $\mathrm{e}_{1}$. Pour + inf. exige au contraire que le contrôleur du procès à l'infinitif $\mathrm{e}_{2}$ soit co-référent avec le sujet du procès $\mathrm{e}_{1}$ : il ne pourra donc traduire les occurrences tant du $\mathrm{CO}$ que de $d a+$ potentiel dans lesquelles il y a non-coréférence entre le sujet de $\mathrm{e}_{1}$ et celui de $\mathrm{e}_{2}$.

(vii) pour + inf., nous l'avons vu également, affecte dans $4 \%$ des cas des événements présents ou futurs. Ces occurrences-là, comme p. ex. [32-33], ne sont transposables à $d a+$ potentiel, ni non plus au CO.

Le CO, forme la moins contrainte, ce qui explique qu'il puisse transposer fréquemment les tours NT, ne peut cependant pas toujours leur proposer une équivalence, comme l'explicite (viii) :

(viii) Outre que, comme vu supra (vii), le CO ne puisse pas transposer un pour + inf. actualisant des événements présents ou futurs, il n'est pas compatible avec des adverbes ou des circonstants temporels déictiques, même s'ils situent lesdits événements dans le passé. Soit l'occurrence NT serbe [39] :
[39] Takva je dozvola najpre
data prošle subote, $\boldsymbol{d} \boldsymbol{a} \boldsymbol{b i}$ ubrzo
bila povučena na sugestiju vlade. (Tanjug, 12.06.1996.)
Telle est permission d'abord
autorisée samedi dernier, que fut bientôt été retirée
à la suggestion du gouvernement.
Une telle permission a été d'abord autorisée samedi dernier, pour être bientôt retirée à la suggestion du gouvernement.

S'il est possible de la traduire par une NT en français :

[39a] Une telle permission a été d'abord autorisée samedi dernier, pour être bientôt retirée à la suggestion du gouvernement.

on ne saurait la traduire par un $\mathrm{CO}$ :

[39b] *Une telle permission a été d'abord autorisée samedi dernier ; elle serait bientôt retirée à la suggestion du gouvernement.

Ce que nous expliquons par le fait que le conditionnel objectif actualise $\mathrm{e}_{2}$ comme ultérieur anaphoriquement à partir de $\mathrm{e}_{1}$. La langue (au moins pour l'heure) rechigne à doubler ce calcul d'ultériorité anaphorique d'un ancrage d'antériorité déictique (samedi dernier). 


\section{Conclusion}

Nous sommes partis de l'observation que les langues française et serbe ont trouvé des constructions pour signifier explicitement la relation de progression temporelle effective entre un événement $\mathrm{e}_{1}$ et un événement $\mathrm{e}_{2}$ dans le passé, soit $\left[\mathrm{e}_{1}<\mathrm{e}_{2}\right]: \mathrm{e}_{2}$ peut être actualisé au conditionnel objectif en français, ou intégré à une proposition subordonnée non téléonomique en français et en serbe.

Ces constructions se sont développées en grammaticalisation de tours antérieurs: le conditionnel subjectif pour le tour $\mathrm{CO}$, la subordination téléonomique pour les NT. L'opération de grammaticalisation s'est réalisée sur une longue durée: les premières occurrences de $\mathrm{CO}$ datent du début du XIX ${ }^{\mathrm{e}}$, celles de NT en français du XVII ${ }^{\mathrm{e}}$; nous ne sommes pas à même actuellement de dater les premières occurrences de NT en serbe : on fait pour l'heure l'hypothèse que le tour est récent, dans la mesure où les grammaires n'en font pas mention.

Nous avons décrit le fonctionnement de ces trois constructions à partir d'un corpus d'une centaine d'occurrences pour chacune d'elles, calculé le taux de transpositions possibles de l'une à l'autre, puis analysé les raisons qui bloquent les transferts : elles tiennent à la structure de chacune de ces constructions et aux contraintes d'ordre syntaxique, sémantique et énonciatif qu'elle leur impose. Il est apparu que certaines de ces contraintes pouvaient être comprises comme des rémanences du tour originel dont chacune des trois constructions procède.

Nous n'avons pas analysé l'effet de sens qu'elles produisent en discours : dans les trois cas, sans que l'on puisse noter de différences, il s'agit d'une forte focalisation sur l'événement $e_{2}$, focalisation qui procède de ce que $\mathrm{e}_{2}$ est mis en perspective d'ultériorité à partir de $\mathrm{e}_{1}$.

Pour finir, disons que ces constructions sont d'un emploi sporadique : si elles font partie de la pratique scripturale des journalistes et des romanciers, elles ne sont nullement d'un usage fréquent. Il semble d'autre part qu'elles soient pour l'heure cantonnées à l'écrit: nos corpus ne comportaient pas d'occurrence procédant d'une interaction conversationnelle. Signalons toutefois qu'une recherche complémentaire nous a permis d'en «dénicher » une dans la base CLAPI, que nous rapportons :

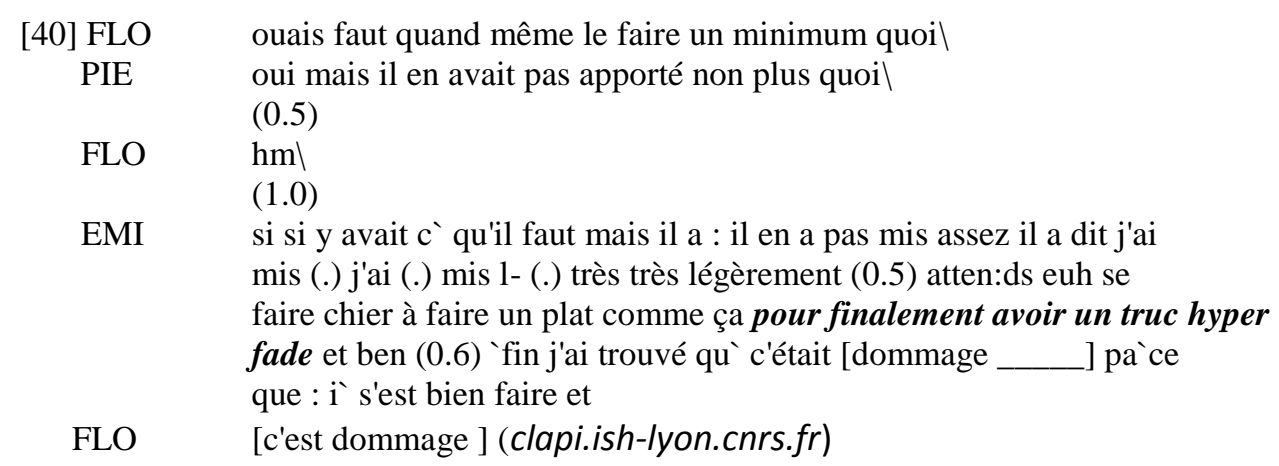


Serait-ce la preuve que, au moins pour ce qui est du français, la construction NT est peut-être en train d'entrer dans l'usage courant?

\section{Références bibliographiques}

ARAÚJo S.-L.-G., 2009, «Sobre a natureza composicional da categoria aspecto : do plano nocional ao plano enunciativo », Diacrítica, 23(1), p. 23-54.

BENVENISTE E., 1966 [1959], «Les relations de temps dans le verbe français », Problèmes de linguistique générale, Paris, Gallimard, p. 237-257.

BRES J., 2010, «Arthur, où t'as mis ton corps ? Conditionnel et actualisation de l'effet de sens historique », in Bres, J., Arabyan, M., Ponchon, Th., Rosier, L., Tremblay, R., et Vachon-l'HeureuX, P., Actes du colloque international de l'AIPL, Le concept d'actualisation en psychomécanique, Bruxelles, Limoges, Lambert Lucas, p. 193-204.

BRES J., 2012, «Conditionnel et ultériorité dans le PASSÉ : de la subjectivité à l'objectivité », Congrès Mondial de Linguistique Française (CMLF), Lyon, 4-7 juillet, http ://www.ilf-cnrs.fr/ p. 1719-1730.

BURIDANT C., 2001, Grammaire nouvelle de l'ancien français, SEDES.

CADIOT P., 1991. La préposition pour, Paris, Presses du CNRS.

CADIOT P., 1997, Les prépositions abstraites en français, Paris, Armand Colin.

Colinet M., Danlos L., Dargnat, M., Winterstein G., 2014, «Emplois de la préposition pour suivie d'une infinitive : description, critères formels et annotation en corpus », in NEVEU F. et al., Actes du Congrès Mondial de Linguistique française, p. 3041-3058.

Costa M.-L., 2010, « Entre a "noção lexical" e a "noção gramatical" : hibridismo nocional das preposições », in CORREIA C.-N., WGT - Formas \& Construções, Centro de Linguistica da Universidade Nova de Lisboa, p. 4351.

DAmourette J. et Pichon E., 1911-1936, Des mots à la pensée, Paris, d'Artrey.

DIVJAK D., 2001, "On the expression of purpose in Russian: augmented teleonomic versus čtobyconstructions », Slavica Gandensia, 28, p. 21-49.

GARDIES J.-L., 1975, La logique du temps, Paris, PUF.

MARTIN R., 1971, Temps et aspect. Essai sur l'emploi des temps narratifs en moyen français, Paris, Klincksieck.

MOIGNET G., 1988, Grammaire de l'ancien français, Paris, Klincksieck.

Nilsson EhLE H., 1943, «Le conditionnel "futur du passé" et la périphrase devait + infinitif », Studia neophilologica, 16, p. 50-88.

POPOVIĆ, Lj., 1977, « Namerne rečenice u funkciji naporednih », Književnost i jezik, 24/1, p. 154-163. 
Auteurs : *AŠÍć Tijana, Faculté des Lettres et des Arts, Université de Kragujevac, Jovana Cvijića bb, 34000, Kragujevac (Serbie), Faculté de Philologie, Université de Belgrade, Studentski trg 3, 11000, Belgrade (Serbie).

tijana.asic@gmail.com

*BRES Jacques, Praxiling, UMR 5267, Université Montpellier 3, Route de Mende, 34090, Montpellier (France)

jacques.bres@univ-montp3.fr

*Dodig Milana, Faculté des Lettres et des Arts, Université de Kragujevac, Jovana Cvijića bb, 34000, Kragujevac (Serbie)

dodigmilana@ filum.kg.ac.rs

*Torterat Frédéric, LIRDEF, EA 3749, Université de Montpellier, Faculté d'Education, 2 Place Marcel Godechot, 34092 Montpellier Cedex 5 (France)

frederic.torterat@umontpellier.fr 